\title{
Auditory stimulus-response modeling with a match-mismatch task
}

De Cheveigné, Alain; Slaney, Malcolm; Fuglsang, S. ren A.; Hjortkjaer, Jens

Published in:

Journal of Neural Engineering

Link to article, DOI:

10.1088/1741-2552/abf771

Publication date:

2021

Document Version

Publisher's PDF, also known as Version of record

Link back to DTU Orbit

Citation (APA):

De Cheveigné, A., Slaney, M., Fuglsang, S. R. A., \& Hjortkjaer, J. (2021). Auditory stimulus-response modeling with a match-mismatch task. Journal of Neural Engineering, 18(4), [046040]. https://doi.org/10.1088/17412552/abf771

\section{General rights}

Copyright and moral rights for the publications made accessible in the public portal are retained by the authors and/or other copyright owners and it is a condition of accessing publications that users recognise and abide by the legal requirements associated with these rights.

- Users may download and print one copy of any publication from the public portal for the purpose of private study or research.

- You may not further distribute the material or use it for any profit-making activity or commercial gain

- You may freely distribute the URL identifying the publication in the public portal

If you believe that this document breaches copyright please contact us providing details, and we will remove access to the work immediately and investigate your claim 
PAPER • OPEN ACCESS

\section{Auditory stimulus-response modeling with a match-mismatch task}

To cite this article: Alain de Cheveigné et al 2021 J. Neural Eng. 18046040

View the article online for updates and enhancements.
You may also like

\begin{tabular}{l} 
- A novel beamformer-based imaging of \\
$\frac{\text { phase-amplitude coupling (BIPAC) }}{\text { unveiling the inter-regional connectivity of }}$ \\
$\frac{\text { emotional prosody processing in women }}{\text { with primary dysmenorrhea }}$ \\
Hui-Ling Chan, Intan Low, Li-Fen Chen et \\
al. \\
- Electrophysiology of the auditory system \\
\hline P J Abbas \\
- EEG can predict speech intelligibility \\
Ivan lotzov and Lucas C Parra
\end{tabular}

\section{FLS1000}

PHOTOLUMINESCENCE SPECTROMETER 


\title{
Journal of Neural Engineering
}

PAPER

CrossMark

OPEN ACCESS

RECEIVED

5 November 2020

REVISED

18 March 2021

ACCEPTED FOR PUBLICATION

13 April 2021

PUBLISHED

4 May 2021

Original Content from

this work may be used

under the terms of the

Creative Commons

Attribution 4.0 licence.

Any further distribution

of this work must

maintain attribution to

the author(s) and the title

of the work, journal

citation and DOI.

\section{Auditory stimulus-response modeling with a match-mismatch task}

\author{
Alain de Cheveigné ${ }^{1,2,3,7, *}\left(\mathbb{C}\right.$, Malcolm Slaney ${ }^{4}\left(\mathbb{C}\right.$, Søren A Fuglsang ${ }^{6}$ and Jens Hjortkjaer ${ }^{5,6}$ \\ 1 Laboratoire des Systèmes Perceptifs, Paris, CNRS UMR 8248, France \\ 2 Département d'Etudes Cognitives, Ecole Normale Supérieure, Paris, PSL, France \\ 3 UCL Ear Institute, London, United Kingdom \\ 4 Google Research, Machine Hearing Group, Mountain View, CA, United States of America \\ 5 Hearing Systems Section, Department of Health Technology, Technical University of Denmark, Kgs. Lyngby, Denmark \\ 6 Danish Research Centre for Magnetic Resonance, Centre for Functional and Diagnostic Imaging and Research, Copenhagen University \\ Hospital Hvidovre, Copenhagen, Denmark \\ 7 Audition, DEC, ENS, 29 rue d'Ulm, 75230 Paris, France \\ * Author to whom any correspondence should be addressed. \\ E-mail: Alain.de.Cheveigne@ens.fr
}

Keywords: EEG, MEG, BCI, auditory, decoding, CCA, attention decoding

\begin{abstract}
Objective. An auditory stimulus can be related to the brain response that it evokes by a stimulus-response model fit to the data. This offers insight into perceptual processes within the brain and is also of potential use for devices such as brain computer interfaces (BCIs). The quality of the model can be quantified by measuring the fit with a regression problem, or by applying it to a classification task and measuring its performance. Approach. Here we focus on a match-mismatch (MM) task that entails deciding whether a segment of brain signal matches, via a model, the auditory stimulus that evoked it. Main results. Using these metrics, we describe a range of models of increasing complexity that we compare to methods in the literature, showing state-of-the-art performance. We document in detail one particular implementation, calibrated on a publicly-available database, that can serve as a robust reference to evaluate future developments. Significance. The MM task allows stimulus-response models to be evaluated in the limit of very high model accuracy, making it an attractive alternative to the more commonly used task of auditory attention detection. The MM task does not require class labels, so it is immune to mislabeling, and it is applicable to data recorded in listening scenarios with only one sound source, thus it is cheap to obtain large quantities of training and testing data. Performance metrics from this task, associated with regression accuracy, provide complementary insights into the relation between stimulus and response, as well as information about discriminatory power directly applicable to BCI applications.
\end{abstract}

\section{Introduction}

Continuous stimuli such as speech or music elicit an ongoing brain response (Ahissar et al 2001, Aiken and Picton 2008, Power et al 2011, Ding and Simon 2012, Kubanek et al 2013) that can be detected with electroencephalography (EEG) or magnetoencephalography (MEG). The relation between stimulus and response can be characterized by fitting a model to the data (Lalor et al 2009, Crosse et al 2016). Most work has used a linear stimulus-response model to relate some feature transform of the stimulus (envelope, spectrogram, etc) to the brain response. Such models come in three main flavors: a forward model that attempts to predict the neural response from the stimulus (Lalor et al 2009, Ding and Simon 2012, Crosse et al 2016), a backward model that attempts to infer the stimulus from the response (Mesgarani and Chang 2012, O'Sullivan et al 2015, 2019, Puvvada and Simon 2017, Hausfeld et al 2018, Akbari et al 2019), or a hybrid forward-backward model that transforms both stimulus and response to better reveal their relation (Dmochowski et al 2017, de Cheveigné et al 2018, Zhuang et al 2020). The fit of these models is usually quantified by calculating the correlation coefficient between the true and predicted responses: the observation of a significant correlation suggests that the model captures some aspect of neural processing, and details of the model (e.g. latency or shape of a temporal response function) then provide insights into 
the sensory processing mechanisms at work within the brain.

A stimulus-response model can also be applied to a classification task, and its quality evaluated based on performance in that task. Auditory attention decoding (AAD) has played an important role in past studies (Kerlin et al 2010, Power et al 2011, Ding and Simon 2012, Mesgarani and Chang 2012). A subject is instructed to attend to one of two concurrent streams, usually speech, and the algorithm decides which stream was attended based on the brain activity (figure 1, left). Model accuracy can be quantified by classification performance (for example percent correct), and the task itself may be relevant for applications such as controlling a device (for example a hearing aid) based on which stream is the focus of attention. However, AAD requires a two-voice stimulus, specific instructions to subjects, and a wellcontrolled experimental setup. Data for training and evaluation depend on labels defined by the experimental task (specifically which voice the subject is attending). The listener's attentional state may stray momentarily from instructions (e.g. attentional capture by the 'unattended' stream) and so some proportion of the data may be mislabeled. This can be a problem if we wish to evaluate algorithms in the limit of small error rates.

In this paper, we consider a different classification task (match-mismatch, MM) that applies to listening scenarios with only one sound source. This task consists of deciding whether a segment of EEG or MEG is temporally aligned with a segment of audio (i.e. that segment of response was evoked by that particular segment of stimulus), or not (figure 1, right). Compared to $\mathrm{AAD}$, the MM task offers a simpler and potentially more efficient framework to optimize stimulus-response models. It can be applied in listening scenarios where there is only one speaker, and does not depend on whether the listener followed instructions as to which stream to attend (variations in attention to the single stream are possible but less disruptive). As no data labels are required, models may be trained for this task in a self-supervised manner. This simpler task is applicable to the evaluation of high performance algorithms with small error rates. To the extent that both $\mathrm{MM}$ and $\mathrm{AAD}$ rely on the accuracy of the stimulus-response models, we speculate that models optimized with one may yield improved performance on the other. With an MM model, performance can be quantified by either the sensitivity index, defined here as the standardized mean of the distribution of the decision metric, or the error rate. Together, correlation, sensitivity index, and error rate form a trio of complementary performance metrics of stimulus-response models.

Building on prior work, cited above, we introduce a set of refinements applicable to a stimulus-response model and evaluate them within the MM task framework. These refinements allow more complex models while controlling for overfitting. As we will show, error rates averaged over subjects for $5 \mathrm{~s}$ segments fall from $\sim 30 \%$ for our simplest model to $\sim 3 \%$ for the best $(0 \%$ error for a subset of subjects) indicating considerably more reliable stimulusresponse models. We devote effort to understanding which processing steps improve performance, and why. In the past, progress has been slowed by the lack of reliable comparative evaluation due to the diversity of experimental conditions and data, the absence of state-of-the-art algorithms in the 'line-up', and the aforementioned issues with associated with the AAD task. We use a publicly available database, metrics based on the simpler MM task, and a well-defined implementation of a competitive method to facilitate evaluation of future advances.

This study offers two main contributions. First, it introduces a simple objective task, match-mismatch (MM), to help in the evaluation of stimulus-response models. Second, it documents a set of techniques that boost performance beyond state of the art.

\section{Methods}

This section describes the stimulus-response model and provides details of the evaluation methods and experiments. The busy reader is encouraged to read the section 2.1, then skip to section 3, Results, and come back for more details as needed. We assume that brain responses are recorded by EEG, but the same methods are applicable to MEG or other recording modalities.

\subsection{Models and metrics}

In this subsection we define the mathematical tools to describe what we wish to accomplish, and the metrics to judge success.

\subsubsection{Data model}

The brain response data consist of a time series matrix $\mathbf{X}$ of dimensions $T$ (time) $\times J$ (channels). Each channel is the weighted sum of brain sources of interest as well as undesired noise and artifacts:

$$
x_{j}(t)=\sum_{i} s_{i}(t) m_{i j},
$$

where $t$ is time, $\left[s_{i}(t)\right], i=1 \ldots I$ are sources, and the $m_{i j}$ are unknown source-to-sensor mixing weights. In matrix notation $\mathbf{X}=\mathbf{S M}$. This matches the physical source-to-sensor mixing process which is, to a good approximation, linear and instantaneous. The audio stimulus is represented as a matrix or column vector A, usually a transform such as the waveform envelope (akin to a measure of 'instantaneous loudness') or the spectrogram (akin to an 'auditory nerve activity pattern'). $\mathbf{A}$ is of size $T \times K$, where $K$ is the number of channels of the stimulus representation (e.g. number of frequency bands of a spectrogram). In the following, $K=1$. 
AAD task:

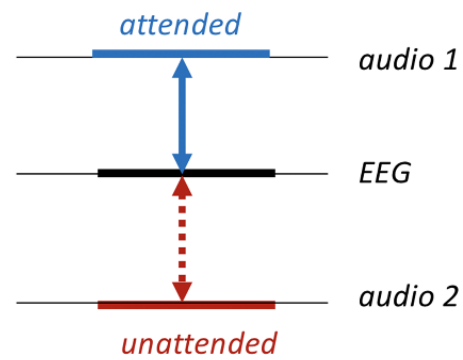

MM task:

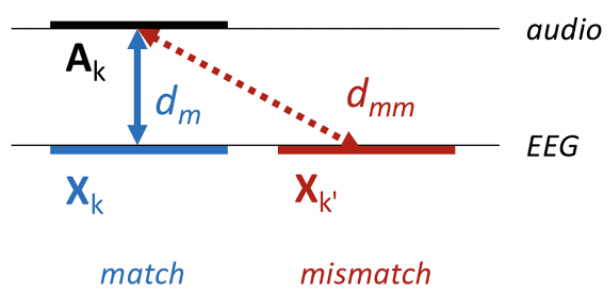

Figure 1. Left: the classic auditory attention detection task (AAD). A segment of EEG data is compared, via a model, to two audio streams, one attended and the other unattended. Right: the new match-mismatch task (MM). A segment of audio is compared, via a model, to the segment of EEG that it evoked (match, Euclidean distance $d_{m}$ ) or some unrelated segment (mismatch, $\left.d_{\mathrm{mm}}\right)$.

\subsubsection{Stimulus-response model}

We assume that a transform $\mathbf{F}$ of the stimulus representation is non-trivially related to a transform $\mathbf{G}$ of the EEG:

$$
\mathrm{AF} \approx \mathrm{XG}
$$

where $\approx$ indicates similarity according to some metric. By non-trivial we mean that equation (2) can be used empirically to decide whether or not a segment $\mathbf{X}_{s}$ of the brain data was recorded in response to a segment $\mathbf{A}_{\mathbf{s}}$ of the stimulus. $\mathbf{F}$ and $\mathbf{G}$ are linear transform matrices (possibly convolutive), but equation (2) can be usefully generalized to more complex transforms, e.g. $f_{X}(\mathbf{A}) \approx g_{A}(\mathbf{X})$.

Three special cases are worth noting. In the forward model, $\mathbf{A F} \approx \mathbf{X}$, the transform $\mathbf{F}$ is used to predict the response from the stimulus. In the backward model, $\mathbf{A} \approx \mathbf{X G}$, the transform $\mathbf{G}$ is used to infer the stimulus from the response. Forward and backward models are also referred to as 'encoding' and 'decoding' (Naselaris et al 2011), or 'temporal response function' (TRF) and 'stimulus reconstruction' models, respectively. A third hybrid model involves transforms of both: $\mathbf{A F} \approx \mathbf{X G}$. Tradeoffs between these three approaches are reviewed in the section 4 .

The transforms $\mathbf{F}$ and/or $\mathbf{G}$ are found by a datadriven algorithm, regression for the first and second models, or canonical correlation analysis (CCA) for the third. Given datasets A and X, CCA finds transforms such that (a) columns of AF are orthonormal (variance 1 and mutually uncorrelated), (b) columns of XG are orthonormal, (c) the pair formed by the first column of $\mathbf{A F}$ and the first column of $\mathbf{X G}$ has the greatest possible correlation on the training data, the pair formed by the second columns has the greatest correlation once the first columns have been projected out, and so-on. Matrices $\mathbf{F}$ and $\mathbf{G}$ are of size $J \times H$ and $K \times H$ respectively, where $H$ is at most equal to the smaller of $J$ and $K$.

\subsubsection{The match-mismatch task}

To assist evaluation, we define the match-mismatch (MM) task as follows. Given a segment of stimulus signal $\mathbf{A}_{\mathbf{s}}$, the segment of EEG signal $\mathbf{X}_{\mathbf{s}}$ that it evoked, and some unrelated segment of EEG signal $\mathbf{X}_{\mathbf{s}^{\prime} \neq \mathbf{s}}$, decide which of the two EEG segments matches, via a model, the stimulus (figure 1, right). A practical application might be to determine whether a user is attentive to sound, or whether a particular alarm sound was noticed. Here we use it simply to measure the accuracy of the stimulus-response model.

\subsubsection{Metrics}

Goodness-of-fit will be evaluated using three metrics: correlation, sensitivity index, and classification error rate, the last two contingent on the MM task. The first, correlation, is calculated between transforms $\mathbf{A F}$ and $\mathbf{X G}$ over the full duration of the data, or over a shorter segment of duration $D$. When the transformed data are normalized, as they are in this paper, correlation is related to Euclidean distance by the relation $r=1-d^{2} / 2$. A perfect match is characterized by $r=1, d=0$ and lack of correlation by (in expectation) $r=0$, $d=\sqrt{2}$.

The second metric, sensitivity index, is based on the distribution of the difference $\Delta_{s}=d_{\mathrm{mm}}-d_{m}$ of Euclidean distances for matched and mismatched segments. For each segment $s$ of stimulus (transformed and $\mathrm{z}$-scored), $d_{\mathrm{mm}}$ is calculated as the average distance to mismatched segments $s^{\prime}$ of EEG (transformed and z-scored), over all $s^{\prime} \neq s$, while $d_{m}$ is the distance to the matched segment of EEG features. Values of $d_{\mathrm{mm}}$ cluster around $\sqrt{2}$ because the data are normalized and mismatched segments are uncorrelated. Matched distances $d_{m}$ tend to have smaller values, and so the difference $\Delta_{s}$ is (hopefully) positively distributed. The sensitivity index is calculated as the mean of this distribution divided by its standard deviation (standardized mean):

$$
z=m / \sigma
$$

This definition is analogous to that of the 'standardized mean difference' (d-prime), but differs in that it quantifies the distribution of the difference between $d_{m}$ and $d_{\mathrm{mm}}$, rather than the distributions of those values 
themselves. For CCA, the distances are calculated taking into account the first 5 CCs with equal weight. In a previous study (de Cheveigné et al 2018) we used linear discriminant analysis to optimally combine CCs, here we found less benefit so we report only this simpler scheme.

The third metric, error rate, counts the proportion of segments classified incorrectly in the MM task (the proportion of segments $s$ for which $\Delta_{s}<0$ ). Sensitivity and error rate depend on segment duration $D$, which is varied as a parameter: the shorter the segment, the noisier the correlation or decision calculation, and the harder the task. Error rate $(e)$ is preferred to proportion correct $(1-e)$ because, plotted on a logarithmic scale, it better reveals incremental steps towards better performance, particularly in the region of high performance. Each metric has its virtues, as elaborated in section 4, Discussion.

\subsubsection{Cross-validation}

Regression and CCA are data-driven and thus prone to overfitting. To avoid overly optimistic results, a model can be trained and tested on different sets of data, for example using $\mathcal{K}$-fold cross-validation (Murphy 2021). Data are divided into $\mathcal{K}$ trials, the model is fit on $\mathcal{K}-1$ trials and performance metrics are evaluated on the $k$ th (left-one-out), the final score being the average of these $\mathcal{K}$ estimates.

\subsection{Extending and reducing the model}

At least three factors degrade the model fit: latency, spectral mismatch between the stimulus representation and the brain response, and additive noise in the response. These can be addressed in part by augmenting the data with a set of time lags (or a filter bank).

\subsubsection{Lags and time shift}

Brain responses unfold over time, and there may be a convolutive mismatch (e.g. delay) in the process connecting stimulus and response. These can be absorbed by augmenting the stimulus and/or brain signals with time lags. Applying a set of lags $0 \ldots L_{A}-1$ to $\mathbf{A}$ and concatenating the time-lagged channels side by side yields a matrix of size $T \times K L_{A}$. Similarly, applying $L_{X}$ lags to $\mathbf{X}$ yields a time-lagged matrix of size $T \times J L_{X}$. An appeal of lags is that they allow the algorithm (univariate regression or CCA) to automatically synthesize a finite impulse response filter (FIR) or, in the case of multichannel data, a multichannel FIR. This allows the model to minimize spectral mismatch (amplitude and phase) between $\mathbf{A}$ and $\mathbf{X}$. The number of lags $L$ determines the order of the synthesized FIR filter. A larger $L$ confers the ability to select or reject temporal patterns on a longer time scale (lower frequencies), at the cost of greater computational cost and greater risk of overfitting.

In addition to lags, we introduce an overall time shift $S$ between stimulus and response. This parameter, distinct from the lags, is intended to absorb any gross temporal mismatch due to instrumental or sensory latencies. This frees the lag parameters to fit finer spectro-temporal characteristics. Without $S$ a larger value of $L$ might be needed, with greater risk of overfitting. $S$ is treated as a hyperparameter: the fit is repeated for several values and the one that yields the highest correlation value is retained.

\subsubsection{Dyadic filter basis}

Lags $0 \ldots L-1$ form a basis of the space of FIR filters of order $L$, but one can choose a different basis, for example outputs of a $L$-channel filter bank of FIRs of order $L$. To reduce dimensionality, one can then choose a subset $L^{\prime}<L$ of that basis, defining a $L^{\prime}$-dimensional subspace of the space of FIRs of order $L$. With a judicious choice of filter bank, performance with $L^{\prime}<L$ channels may be superior to merely choosing $L^{\prime}<L$ lags, in part due to a lower risk of overfitting. For example, a logarithmic filter bank (e.g. wavelet, or dyadic, c.f. https://ccrma.stanford.edu/ jos/sasp/Dyadic_Filter_ Banks.html) can capture patterns of both short and long time scale with a limited number of channels, whereas capturing the same long time scale with a basis of lags would entail a much larger dimensionality. Here, we use a dyadic filter basis.

\subsubsection{Dimensionality reduction}

The models we describe here can be large, including a large number of parameters, leading to overfitting if we do not have enough training data. Overfitting can be made less severe by reducing the dimensionality of the data before fitting the model, or by applying regularization within the fitting algorithm (Wong et al 2018). The two approaches are closely related (Tibshirani et al 2017, section 3.4.1). Here, we use dimensionality reduction. Data are submitted to principal component analysis (PCA) and principal component (PCs) beyond a certain rank $N$ are discarded, thus ignoring directions of low variance within the data. Ridge regularization similarly shrinks low-variance directions (Tibshirani et al 2017).

\subsection{Evaluation}

Given the task described above, there are several ways we can measure success. This subsection describes the methodology, using cross-validation to measure success.

\subsubsection{Data}

The data we use here are from a study that aimed to characterize cortical responses to speech for both normal-hearing and hearing-impaired listeners (Fuglsang et al 2020). Experimental details are provided in that paper and the data are publicly available. In brief, 64-channel EEG responses to acoustic stimuli (audiobook) were recorded at a sampling rate of $512 \mathrm{~Hz}$ from 44 subjects, including both normal-and hearing-impaired. Stimuli for the latter 


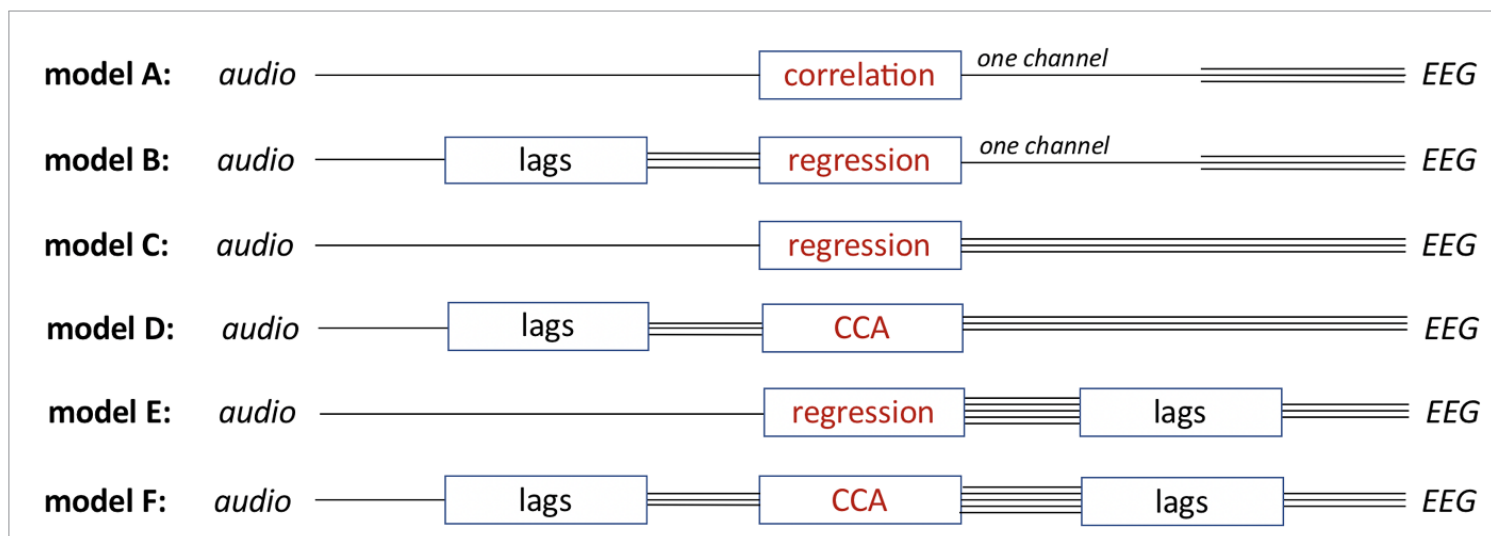

Figure 2. The six basic stimulus-response models considered in this study.

were equalized (frequency-specific amplitude boost) to compensate for the impairment, and we pool data from both. Including both populations results in a larger and more diverse data set, with results possibly valid for a wider population (some applications target impaired users). Stimuli presented to each subject included 16 segments of single-talker speech with a male or female talker speaking in quiet, each of $50 \mathrm{~s}$ duration, that we consider in this study. Other stimuli presented in the same recording session (concurrent speech, tones) are not used. The publicly available dataset includes the temporal envelope of the speech stimulus, sampled at the same rate as the EEG, calculated by a model of instantaneous loudness that has been shown to be a predictor of cortical responses (Lalor et al 2009, Ding and Simon 2012, Di Liberto et al 2015, Crosse et al 2016).

\subsubsection{Preprocessing}

The EEG data were smoothed by convolution with a square window of duration $1 / 50 \mathrm{~Hz}$ (implemented with interpolation) to suppress the line artifact ( $50 \mathrm{~Hz}$ and harmonics) and downsampled by smoothing with a 4-sample square window and decimation by a factor of 4 , to $128 \mathrm{~Hz}$. The data were detrended by applying a robust detrending algorithm (de Cheveigné and Arzounian 2018) that robustly fit a 2nd order polynomial to overlapping intervals of size $15 \mathrm{~s}$, subtracted the fit, and 'stitched' detrended intervals together with a standard overlap-add procedure. The data were then high-pass filtered at $0.5 \mathrm{~Hz}$ using an order-2 Butterworth filter, then low-pass filtered at $30 \mathrm{~Hz}$ also with an order-2 Butterworth filter, and cut into 16 trials of $50 \mathrm{~s}$ duration. To remove eyeblink artifacts, a temporal mask was derived from the absolute power on a combination of two EOG channels and three frontal channels (F1, F2, Fz). Using this mask as a bias, the DSS algorithm was applied to find a transform maximizing eyblink activity (de Cheveigné and Parra 2014) and the first two components (representing eyeblink artifact) were projected out of the EEG data.
To avoid aggravating the mismatch between stimulus and brain response, the stimulus envelope was filtered using the same high pass and low pass filters as for the EEG. All filters were 'single pass' (causal).

\subsubsection{Basic models}

To ease comparison with other studies, we define six models (figure 2) that illustrate basic processing choices, some of which have been made in prior studies and all of which are useful to understand in detail. For each, an overall time shift $S$ is applied to the stimulus relative to the EEG.

- Model A compares one EEG channel with the stimulus envelope, with no spatial or temporal filtering $\left(L_{A}=1, L_{X}=1\right)$ other than the time shift $S$ common to all models.

- Model B compares one EEG channel with a linear combination of time-lagged envelope signals $\left(L_{A}=11, L_{X}=1\right)$ obtained by regression. This corresponds to a standard forward model as reported in the literature.

- Model C compares the envelope to a linear combination of EEG channels (without lags; $L_{A}=1$, $L_{X}=1$ ) obtained by regression. This is analogous to the basic backward model considered in de Cheveigné et al (2018), or the single-delay model of Hausfeld et al (2018)

- Model D compares linear combinations of timelagged envelope signals with linear combinations of EEG channels $\left(L_{A}=11, L_{X}=1\right)$, obtained by CCA. This is analogous to 'CCA model 1' of de Cheveigné et al (2018).

- Model $\mathbf{E}$ compares the envelope with a linear combination of time-lagged EEG channels $\left(L_{A}=1\right.$, $\left.L_{X}=11\right)$ obtained by regression. This is analogous to the backward model of e.g. Fuglsang et al (2017), or the multiple-delay model of Hausfeld et al (2018).

- Model F compares linear combinations of timelagged envelope signals with linear combinations 

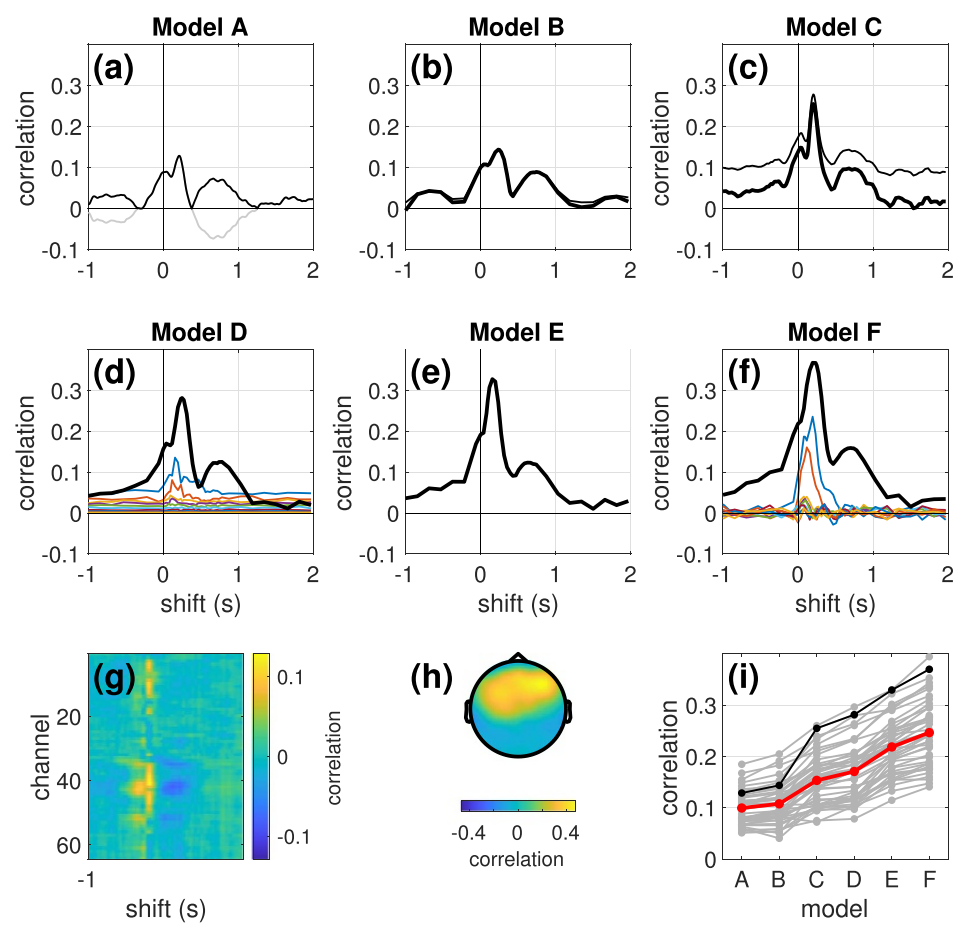

Figure 3. Baseline models A-F for one subject (subject 4, a relatively good subject selected for visual clarity). Panels (a)-(f): correlation as a function of overall time shift $S$ for each model. (a) Cross-correlation function (gray), or absolute value of same (black) between channel 10 of EEG (FC3) and the stimulus envelope (model A). (b) Correlation between channel 10 of EEG and the projection of channel 10 on the time-lagged stimulus, with crossvalidation (thick) or without (thin) (model B). (c)-(f) Cross-validated correlation between stimulus-based and EEG-based components for models C-F. (g) Cross-correlation function between the stimulus envelope and the EEG signal for each EEG channel (as in model A). (h) Topography of correlation coefficients beween EEG-based component of first CC pair and individual EEG channels (model D). (i) Best correlation obtained for each model, for all subjects (gray). The average over subjects is in red, and the selected subject 4 is in black.

of time-lagged EEG channels $\left(L_{A}=11, L_{X}=11\right)$, obtained by CCA. This is analogous to 'CCA model 2' in de Cheveigné et al (2018).

To summarize the similarities and differences: models A and B relate the stimulus to just one of the $J$ EEG channels. In contrast, all other models relate the stimulus to the ensemble of EEG channels. For models $\mathbf{A}-\mathbf{C}$ and $\mathbf{E}$ the fit is based on univariate regression, and for models $\mathbf{D}$ and $\mathbf{F}$ on a multivariate CCA model. For univariate regression models, the fit is quantified by a single correlation coefficient, and for CCA by as many coefficients as CC pairs (figure 2).

Not counting $S$, the number of parameters in the fit is 1 for model $\mathbf{A}, L_{A}=11$ for model $\mathbf{B}, J=64$ for model $\mathbf{C}, L_{A}+J=55$ for model $\mathbf{D}, J L_{X}=704$ for model E, and $L_{A}+J L_{X}=715$ for model $\mathbf{F}$.

\subsubsection{Model $\mathbf{G}$}

In addition to basic models $\mathbf{A}-\mathbf{F}$, we define a reference or 'gold standard' model G, variant of model F, with a performance close to the best we found, and with a relatively straightforward and precisely defined implementation that can help future studies to document further improvements in performance. Details of this model are given in the Results section.

\subsubsection{Display of results, statistics, implementation}

Results are evaluated using the three metrics described above, and plotted as a function of selected parameters chosen to offer insight. Effects are tested for statistical significance using a non-parametric Wilcoxon signed rank test over subjects. Processing scripts in Matlab make use of the NoiseTools toolbox (http://audition.ens.fr/adc/NoiseTools/). Scripts are available at http://audition.ens.fr/adc/NoiseTools/ src/NoiseTools/EXAMPLES/match_mismatch/.

\section{Results}

In the following, we evaluate and compare the models, focusing on the factors that affect performance. Section 3.1 compares performance of the six basic models (A-F; figure 3), using the correlation metric for simplicity and to allow comparison with prior studies. Section 3.2 then introduces the MM classification task, and explores how sensitivity and error metrics depend on segment duration. Section 3.3 explores the dependency of all three metrics on the number of spatial dimensions (number of channels or principal components) and temporal dimensions (lags or filter channels). Based on this, section 3.4 proposes model $\mathbf{G}$ for use as a comparison point in future studies. Section 3.5 investigates factors that cause the classifier to fail, and section 3.6 summarizes performance across models. 


\subsection{Correlation metric for basic Models}

Figure 3 summarizes results obtained with the basic models. The first and second rows display correlation (calculated over the duration of each trial, $\sim 50 \mathrm{~s}$ ) for models A to F for one subject (subject 4). Figure 3(i) summarizes these results by plotting, for each model, the peak cross-validated correlation averaged over subjects (red) and for individual subjects (gray, black for subject 4).

\subsubsection{Model A}

This is the simplest incarnation of a stimulusresponse model (equation 2), with $\mathbf{F}$ and $\mathbf{G}$ both identity transforms. Figure 3(a) shows correlation (gray) between stimulus and EEG as a function of shift $S$ for the best EEG channel (FC3). This is equivalent to the cross-correlation function between stimulus and response (positive and negative values). All other plots represent correlation of a signal with its projection (positive values only). To ease comparison between model $\mathbf{A}$ and the others, the plot also shows the absolute value of the cross-correlation function in black. The shape of the cross-correlation function differs slightly between electrodes (figure $3(\mathrm{~g})$ ), implying that response properties are not uniform across the brain. Peak absolute correlation is 0.13 for this subject; peak values for other subjects can be read off figure 3(i). For the best subject, a bit less than $4 \%$ of the variance of the best EEG channel is explained by the stimulus via this model.

\subsubsection{Model B}

The same EEG channel is projected onto the subspace spanned by the $L_{A}=11$ time-lagged stimulus signals, yielding weights that define an optimized FIR filter applied to the stimulus. Figure 3(b) shows correlation (thin) and cross-validated correlation (thick) as a function of shift $S$ for the best channel (FC3). Cross-validated correlation differs only slightly from raw correlation (thick versus thin) suggesting minimal overfitting in this simple model. Peak correlation is greater than for model A, suggesting that the FIR filter has improved the fit. This improvement is robust across subjects (figure 3(i)), as confirmed by a Wilcoxon signed rank test $\left(p<10^{-8}\right)$.

\subsubsection{Model C}

The stimulus is projected onto the subspace spanned by the $J=64$ EEG channels, yielding an optimized spatial filter. Peak cross-validated correlation between the stimulus signal and its projection (spatiallyfiltered EEG) is greater than for the previous two models across all subjects (figure $3(\mathrm{c}), p<10^{-8}$ ). The topography associated with the projection (correlation with individual EEG channels) shows a pattern typical of auditory responses (figure $3(\mathrm{~h})$ ).

\subsubsection{Model D}

In this model, the time-lagged stimulus is related to the multichannel EEG using CCA. This results in multiple CC pairs, each quantified by a crossvalidated correlation value (figure $3(\mathrm{~d})$ ). The first CC is plotted in black, subsequent CCs are in color. Each CC is defined by a distinct FIR filter applied to the stimulus, and a distinct spatial filter applied to EEG. Multiple CCs suggest that the stimulusresponse model captures multiple brain sources sensitive to different modulation frequency bands within the stimulus. Peak cross-validated correlation for the first CC is greater than for all previous models across subjects $\left(p<10^{-11}\right)$.

\subsubsection{Model E}

Time lags are applied to all EEG channels (but not the stimulus), resulting in a backward model in which the EEG undergoes both spatial and temporal filtering, analogous to the backward model of e.g. Fuglsang et al (2017). Peak cross-validated correlation is greater than for all previous models across subjects $\left(p<10^{-6}\right)$.

\subsubsection{Model F}

Finally, lags are applied to both stimulus and EEG. Each CC then associates a distinct FIR filter applied to the stimulus with a distinct multichannel FIR filter applied to the EEG. Peak cross-validated correlation is again higher than all previous models across subjects $\left(p<10^{-12}\right)$.

Performance improves from models $\mathbf{A}$ to $\mathbf{F}$ for most subjects (figure 3(i) gray lines). Three features seem to contribute to a better fit: spatial filtering leveraging the multichannel nature of EEG (models $\mathbf{C}-\mathbf{F}$ ), temporal filtering allowed by augmenting the data with time shifts (models B-F), and CCA which optimally relates multivariate representations of both stimulus and response (models $\mathbf{D}$ and $\mathbf{F}$ ). It is worth noting that these models differ also in their number of free parameters, from 1 for model A (not counting shift $S$ ) to 735 for model $\mathbf{F}$ (see section 2). One might speculate that more parameters, rather than any particular feature, is what explains the progression in correlation scores. However, these results were obtained for cross-validated correlation for which overfitting should be detrimental. Thus, it seems that the more complex models genuinely provide a better fit, as confirmed with other metrics, below.

\subsection{Classification-based metrics}

We take the best model in terms of correlation (F), and rate its performance on the MM task in terms of metrics sensitivity and error rate, which are both based on the distribution of the difference $\Delta_{s}=d_{\mathrm{mm}}-d_{m}>0$ between matched and mismatched segments of duration $D$ (section 2).

Figure 4(a) shows the distribution of $\Delta_{s}$ for $D=10 \mathrm{~s}$ (red) and $D=1.25 \mathrm{~s}$ (blue). For longer segments, the distribution includes mostly positive values resulting in correct classification, whereas for shorter durations it includes a greater proportion of 

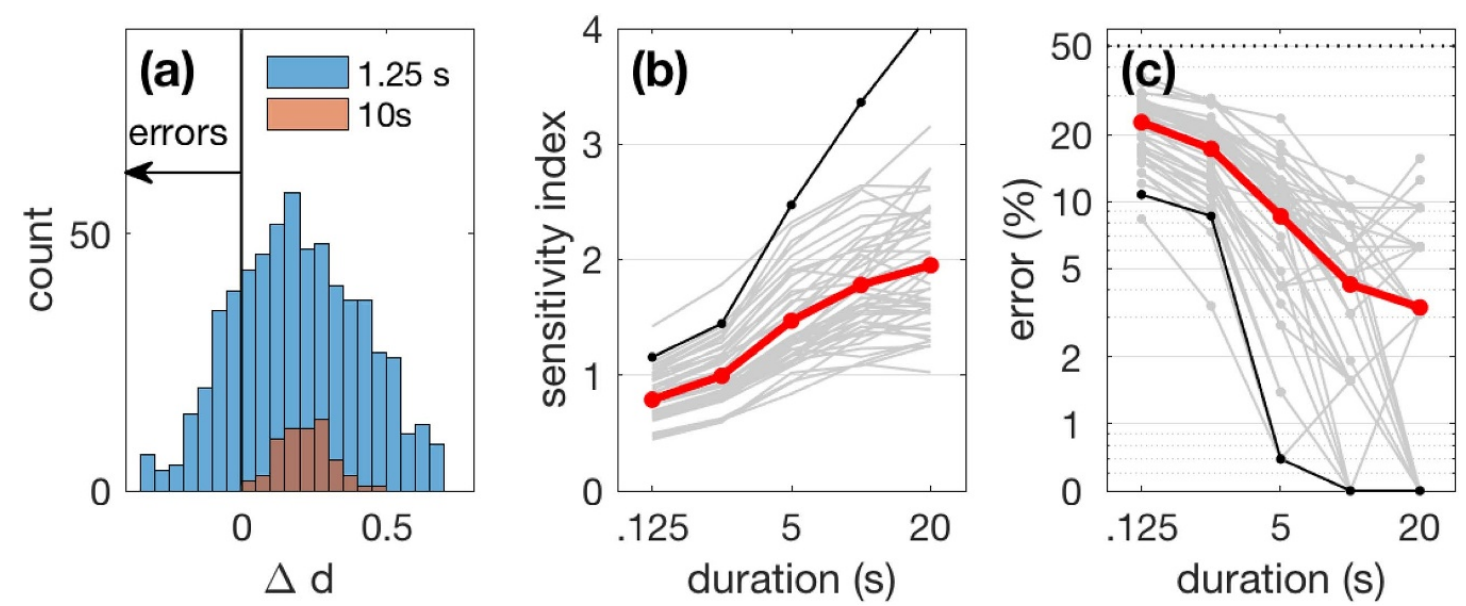

Figure 4. Match-mismatch task. (a) Histogram of $\Delta_{s}$ for segment durations of $1.25 \mathrm{~s}$ (blue) or $10 \mathrm{~s}$ (red), for subject 4 . For shorter segments the histogram is wider and there are more errors $\left(d_{m m}<0\right.$. (b) Sensitivity index $\mu / \sigma$ as a function of segment duration averaged over subjects (red) and for each individual subject (gray, subject 4 is black), for model F. (c) Error rate.
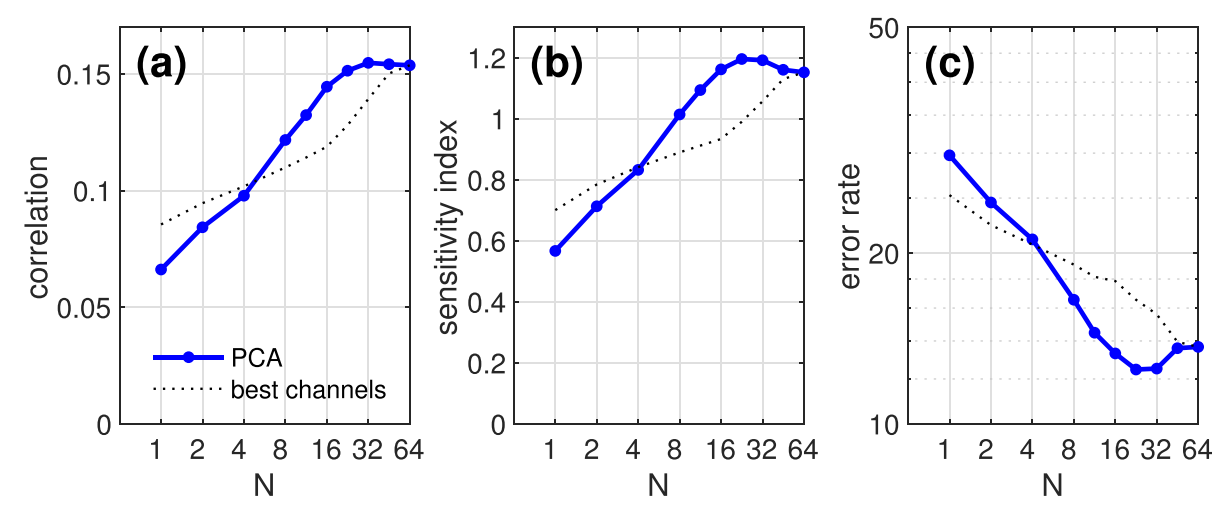

Figure 5. Performance as a function of the number of spatial dimensions, averaged over subjects. (a) Cross-validated correlation as a function of the number of PCs retained after PCA of the 64-channel EEG data. The dotted line represents subect-averaged correlation for subsets of EEG channels chosen for maximal correlation with the stimulus. (b) Sensitivity index. (c) Error rate. The model here includes no lags (similar to model $\mathbf{C}$ ). Segment size is $5 \mathrm{~s}$.

negative values. The degree to which the distribution is dominated by positive values, minimizing error, is captured by the sensitivity index (standardized mean of $\Delta_{s}$ ). Larger is better.

Figure 4(b) shows the sensitivity index as a function of segment duration averaged over subjects (red) and for individual subjects (gray, subject 4 is black). Figure 4(c) likewise shows error rate as a function of duration. We expect the sensitivity index to be greater, and the error smaller, for a longer segment duration because the task is easier (less noise in the correlation estimate), and indeed this is the case. In the following we focus on $D=5 \mathrm{~s}$, for which the error rate averaged over subjects is $\sim 9 \%$ for this model (model F, $L_{A}=$ $L_{X}=11$ ). The variability over subjects is remarkable: at $5 \mathrm{~s}$ the error rate ranges from close to 0 (perfect classification) to more than $20 \%$. Chance rate is $50 \%$.

\subsection{Spatial and temporal dimensionsionality}

This section explores ways to further optimize performance. Comparing basic models (figure 3) it appears that performance can benefit from both spatial filtering and lags. However, a recurring issue for stimulus-response models is overfitting, which depends on the complexity of the model, function here of both the number of spatial dimensions (channels or PCs), and the number of lags. Both factors are explored here.

\subsubsection{Number of spatial dimensions}

Using model C (no lags) as a reference point, figure 5 (blue) shows the effect of applying PCA to the EEG data and discarding PCs beyond a certain rank $N$. The sensitivity index peaks, and the error rate is minimal, for $N \approx 32$, suggesting that overfitting may be occurring due to excess dimensionality and that reducing dimensionality can mitigate its effects.

Truncating the series of PCs is markedly better than the simple expedient of discarding channels (dotted line; channels were sorted by decreasing correlation with the stimulus and the least correlated were discarded). This result is interesting in relation to claims that reducing the number of electrodes can yield equivalent performance to the full set, or even better performance due to less overfitting (Montoya-Martínez et al 2021). Such is not the case 

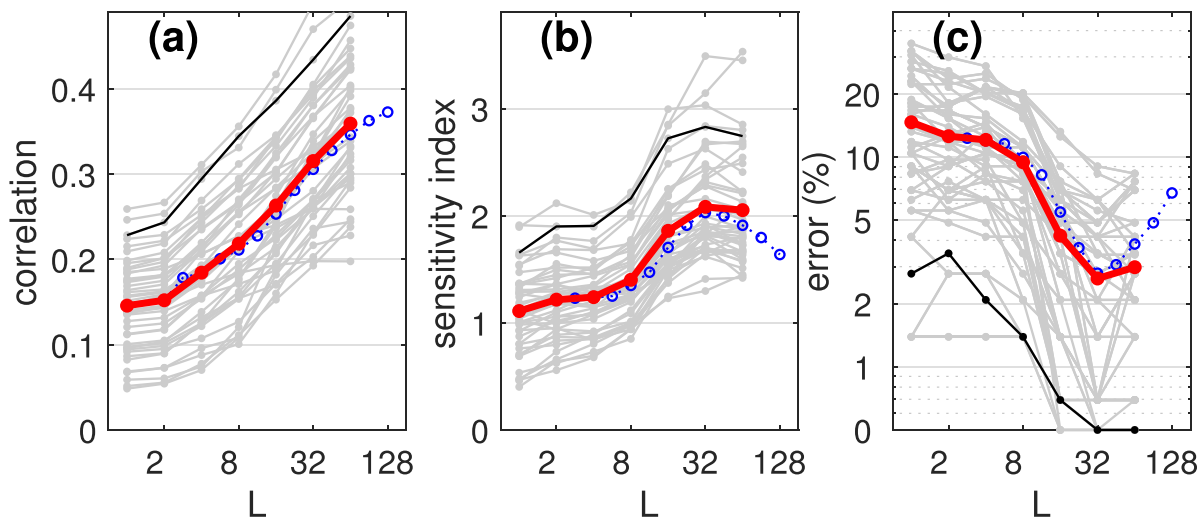

Figure 6. Performance as a function of the number of lags (or the order of the dyadic filters) applied to both stimulus and EEG $\left(L=L_{A}=L_{X}\right)$. (a) Crossvalidated correlation as a function of $L$ averaged over subjects (red) and for all subjects (gray, black is subject 4). Blue symbols are for a dyadic filter bank instead of lags (see text). (b) Sensitivity index. (c) Error rate. Segment size is $5 \mathrm{~s}$.

here: the sensitivity index (figure 5(b), dotted line) rises monotonically, implying that it is best to keep the full set. At no point does performance reach the level that can be attained by selecting PCs from a PCA applied to the full set of electrodes. The conclusion is simple: more electrodes is better.

\subsubsection{Number of lags}

Figure 6 shows metrics of correlation, sensitivity index, and error rate as a function of the number of lags $\left(L=L_{A}=L_{X}\right)$ averaged over subjects (red) and for individual subjects (gray, subject 4 is black). As the number of lags is increased, correlation and sensitivity increase until $\sim L=32(250 \mathrm{~ms})$, then decrease beyond. This peak is mirrored by a dip in error rate at $L=32$. The best error rate is $2.8 \%$ on average over subjects. The reversal beyond $L=32$ might reflect an increase in $d_{\mathrm{mm}}$ relative to $d_{m}$, thus reducing the numerator $m$ in equation (3), or an increase in their variablity, thus increasing the denominator $\sigma$.

A fall in performance with larger $L$ can be a sign of overfitting, as a model with many parameters can fit the minutiae of the training data but generalize poorly to the test data. However, the drop might also have a different cause, for example long lags might capture slow patterns that do not generalize well. We can arbitrate between these explanations by comparing results for lags $1 \ldots L$ (figure 6 , red) with those for a dyadic filter bank with FIR filters of order $L$ (blue). A filterbank of $L^{\prime}$ dyadic filters of order $L$ has fewer channels $\left(L^{\prime}<L\right)$ and thus requires fewer parameters in the model $\left(L^{\prime}=10\right.$ for $L=32 ; L^{\prime}=12$ for $L=64$, etc). Thus, the drop should occur for larger $L$ for the dyadic filterbank (blue) than for lags (red) if it reflected the number of parameters. Instead it occurs at the same value of $L$, suggesting indeed that performance drops because longer lags capture slow patterns that do not generalize well.

In a previous study (de Cheveigné et al 2018) we obtained better performance with the dyadic filterbank than with lags. We do not replicate that result here: performance is similar for both for equal $L$. Once again, the variability of these metrics over subjects is remarkable. For $L=32$, the error rate for five second segments ranges from $0 \%$ for the best ten subjects to $\approx 9 \%$ for the worst. Incidentally, the error rate averaged over hearing-impaired subjects (1.7\%) is smaller than for normal hearing subjects (3.4\%), $p<0.001$, t-test, as was found in other studies (Goossens et al 2018, Decruy et al 2020, Fuglsang et al 2020).

\subsection{Model G ('gold standard')}

Based on these results, we define more precisely one particular model, using one set of parameters that produces close to state-of-the-art performance on a publicly available dataset, to serve as an easyto-implement reference with which to evaluate new algorithms. This reference model and evaluation are described in detail to allow replication.

Model G involves the following steps: (1) a time shift $S$ of $200 \mathrm{~ms}$ is applied to the EEG to advance it relative to the audio envelope, (2) data are preprocessed as described in section 2, (3) PCA is applied to the EEG and the first 32 PCs are selected, (4) audio envelope and EEG are both augmented by applying lags $0 \ldots 31$ (i.e. a $32 / 128=250 \mathrm{~ms}$ range of lags), (5) the augmented data are fit by a linear stimulus/ response model based on CCA, (6) sensitivity and error rate metrics estimated using segments of duration $5 \mathrm{~s}$.

To be precise: for each crossvalidation fold, the CCA solution is trained on a subset of 15 trials and tested on the 16th (left out). All consecutive $5 \mathrm{~s} \mathrm{seg-}$ ments of audio within the left-out trial are considered, and for each the Euclidean distance $d_{m}$ between that segment of audio and the corresponding segment of EEG is calculated (matched distance), as well as the average Euclidean distance $d_{\mathrm{mm}}$ between that audio segment and all EEG segments of all 15 other trials (mismatched distance), yielding a difference 

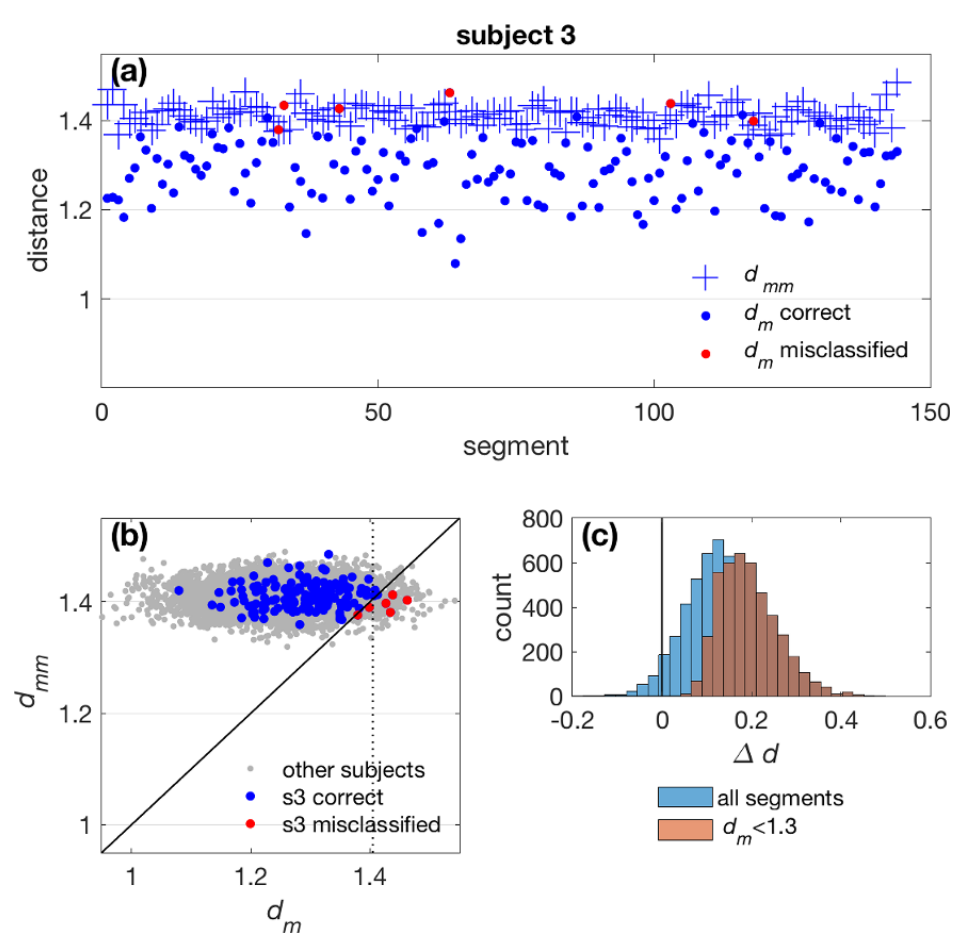

Figure 7. (a) Euclidean distance between matched (dots) and mismatched $(+)$ segments of duration $5 \mathrm{~s}$ for all trials of one subject (subject 3, chosen for a relatively high error rate). Red dots indicate classification errors. (b) Scatterplot of mismatched versus matched distances for subject 3 (blue/red) and all other subjects (gray). The diagonal represents the classification boundary $\Delta_{s}=0$. Points below that line (red) are misclassified. (c) Histograms of values of $\Delta_{s}$ for all segments (blue), and for segments for which the matched distance $d_{m}$ is less than 1.3 (brown).

score $\Delta_{s}=d_{\mathrm{mm}}-d_{m}$ for that segment. Values of $\Delta_{s}$ are aggregated over segments and folds. The ratio between the mean of the distribution of $\Delta_{s}$ and its standard deviation yields the sensitivity metric. The proportion of samples for which $\Delta_{s}$ falls below 0 yields the error rate metric. Distance calculations take into account the first 5 CCs of the CCA solution with equal weights ${ }^{8}$.

To evaluate a new method, the recommended procedure is (1) install model $\mathbf{G}$ on the same system as the new algorithm, (2) test it using the same publicly available database as we use, and verify that the metrics yield scores consistent with what we report, and (3) apply the new method to that database and compare scores with (2). The reason for step (2) is to control for installation-specific differences (e.g. single versus double precision, etc).

Alternatively, if a different database is to be used, do (1) as above, then (2') test model G using the new database, and (3') test the new method on the new database and compare scores with (2'). In any event, it is not recommended to compare a new method with prior methods on a different database, or with different metrics, or with a different task. Thus, there would be little merit in comparing the scores we report here to those reported in the literature for $\mathrm{AAD}$.

\footnotetext{
${ }^{8}$ A Matlab implementation is available at http://audition.ens.fr/ adc/NoiseTools/src/NoiseTools/EXAMPLES/match-mismatch/.
}

\subsection{Anatomy of an error}

One of our goals is to gain a better understanding of factors that determine model performance. An error occurs when the difference $\Delta_{s}=d_{\mathrm{mm}}-d_{m}$ falls below zero, and this might be caused by a relatively small value of $d_{\mathrm{mm}}$ or a relatively large value of $d_{m}$. It is clear from figure 7 that the latter is the main factor for this subject (subject 3, relatively poor model performance). The top panel shows $d_{m}$ (dots) and $d_{\mathrm{mm}}$ (crosses) for all segments of all trials. The mismatched distances are distributed tightly around $d_{\mathrm{mm}} \approx 1.4$ as expected (section 2.1, Metrics) whereas matched distances $d_{m}$ are mostly smaller, as clear also from the scatterplot of $d_{\mathrm{mm}}$ versus $d_{m}$. The diagonal line in figure 7(b) represents the classification boundary $\Delta_{s}=0$ : all points to the right and below this line (red) are misclassified.

The matched distance $d_{m}$ is a good predictor of classification reliablity: for $d_{m}<1.3$ the classification statistic $\Delta_{s}$ is distributed far from the decision boundary (figure 7 (c) brown). Classification is highly reliable in that case, whereas for larger values of $d_{m}$ the classification is less reliable. This implies an asymmetry in the conclusions that can be drawn from the classifier. For example a hypothetical 'attentionmonitoring' device might rapidly and reliably detect that a stimulus has registered within a subject's brain, but the opposite conclusion that it has not registered would take longer and/or be less reliable. Reliability is a useful adjunct to the decision. 


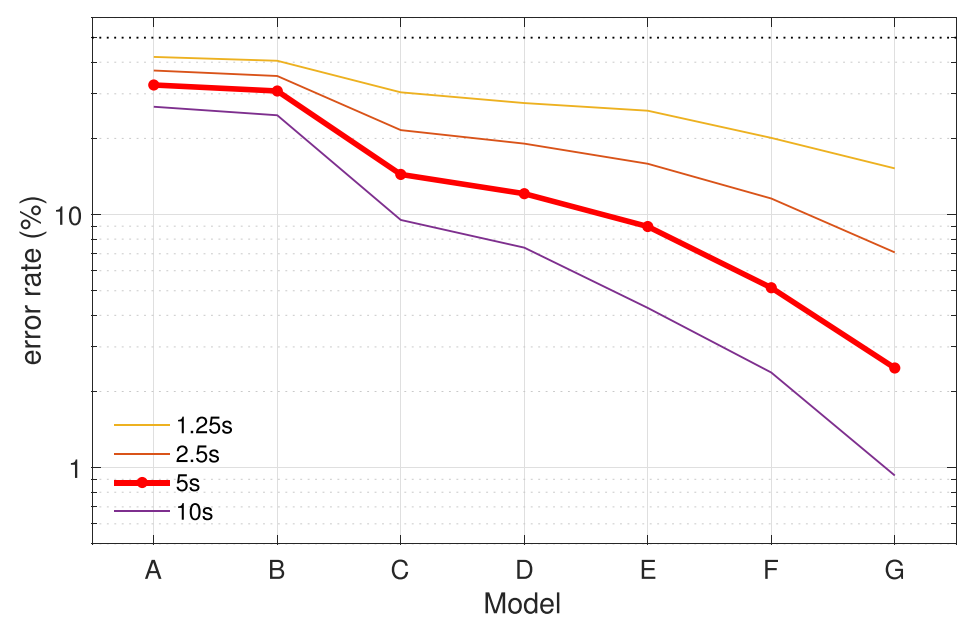

Figure 8. Summary of error rates for models A-G, averaged over subjects, for several values of duration $D$. The dotted line represents chance (50\%). At each duration, model $\mathbf{G}$ makes fewer errors than its closest competitor (F, $p<10^{-4}$, Wilcoxon signed rank test).

What factors might inflate $d_{m}$ ? Regression of d_mm versus the RMS of the EEG signal shows a significant but weak correlation with response power $\left(r=0.12, p<10^{-7}\right)$, suggesting that high-amplitude glitches in the EEG might be a contributory factor. On the other hand, a significant but weak negative correlation with RMS stimulus $\left(r=-0.07, p<10^{-20}\right)$ suggests a possible small contribution of lulls in the stimulus. However, these small correlation values suggest that other factors, unknown, dominate.

\subsection{Summary of methods}

Figure 8 summarizes error rates obtained with each of the models A-G, averaged over subjects. Models $\mathbf{A}$ and $\mathbf{B}$ are classic forward models that attempt to predict one channel of EEG from the stimulus representation. Models $\mathbf{C}$ and $\mathbf{E}$ are classic backward models that attempt to infer the stimulus representation from the EEG. Models D, $\mathbf{F}$ and $\mathbf{G}$ are hybrid models based on CCA. The best model (G) makes an order of magnitude fewer mistakes than the worst (A). For a $5 \mathrm{~s}$ window the error rate for model $\mathbf{G}$ is less than $3 \%$ on average over subjects ( $0 \%$ for 10 subjects). Extrapolating from progress so far, we think that further progress is possible. Associated with the publicly available dataset that we used, model G might serve as a 'gold standard' for comparative evaluation of such future progress.

\section{Discussion}

This study offers two main contributions. First, it introduces a simple objective task to help in the evaluation of stimulus-response models. Second, it documents a set of techniques that boost performance beyond state of the art.

\subsection{The need for an objective task}

A straightforward quality metric for a stimulus-response model is correlation, between response and predicted response in a forward model, between stimulus and inferred stimulus in a backward model, or between transforms of both in a hybrid model. That metric is simple and informative: better models tend to yield higher scores. However an elevated score can also result from chance correlations. These are more widely distributed for data dominated by low frequencies, which could mislead a researcher to conclude that lowpass filtering, for example, improved the model despite the loss of relevant information carried by higher frequencies (Kriegeskorte and Douglas 2019). An objective task alleviates this problem, because loss of relevant information must impair task performance. Another argument in favor of an objective task is that it is a better measure of the model's 'real world' value.

Why three metrics? Firstly, they are not equivalent: referring to figure 6, correlation increases monotonically with lag (a), whereas sensitivity and error rate show a reversal at $L=32(\mathrm{~b}, \mathrm{c})$. Error rate is directly relevant for applications but somewhat coarse and brittle (it depends on a few samples near the classification boundary). Sensitivity depends on all samples by summarizing them based on their mean and standard deviation, but like error rate it requires a task. The appeal of correlation is that it is taskagnostic. Thus, the three metrics are complementary.

\subsection{Selective versus sustained attention}

Auditory attention is often investigated in a situation where multiple stimuli compete for attention, for example concurrent pulse trains (Hillyard et al 1973), or competing voices (Kerlin et al 2010), or competing instruments (Treder et al 2014). It may 
also be probed as a difference in response to a stimulus in the presence, or absence, of concurrent visual stimulation (Molloy et al 2015), or of a behavioral task (Scheer et al 2018). In each case, a comparison is made between brain responses to the same stimulus recorded in two situations. In contrast, the MM task requires only a single recording, and, more importantly, assumes no competition for auditory resources. As such it might be of use to monitor the general attentive (versus inattentive) state of a subject, for example to determine whether an alert has been perceived, or a message has registered, or to detect drowsiness, or evaluate the minimal conscious state in patients in locked-in state, or to detect if a participant is actively paying attention to continue/pause a BCI application.

The AAD task is relevant for BCI applications such as cognitive control of acoustic processing in a hearing aid. However, even for those applications it may be fruitful to optimize the stimulusresponse models using the MM task. We speculate that improvements obtained for the simpler MM task may transfer to the harder AAD task. A practical issue with $\mathrm{AAD}$ is that it relies on specific experimental setups with competing voices, attention task instructions, and greater demands for listening effort. The MM task does not rely on predefined data labels but instead derives them (match versus mismatch) from a manipulation of the input data. It can therefore be applied to any dataset of brain responses to sound. An analogous approach has been used successfully for self-supervised learning, for instance, by training neural networks to predict whether video and audio segments are temporally aligned (Arandjelović and Zisserman 2018, Owens and Efros 2018). The task and metrics are applicable to self-supervised training of large-scale neural networks that require extensive training sets. Being free of reliance on particular 'attention labels', the MM-approach is better suited to evaluate and compare models across datasets with different experimental setups.

Another practical issue with AAD is potential mislabeling due to attentional capture by the wrong stream. We cannot be sure that a subject consistently followed the instructions throughout, and thus a certain proportion of the database might be mislabeled, an important concern when evaluating well-performing models. The MM task is thus appealing as it can be evaluated on data with only one sound stream. A downside is that MM is blind to potential brain processes specific to attention that $\mathrm{AAD}$ might capture. The two tasks are thus complementary.

\subsection{Encoding, decoding, and hybrid models}

A forward (encoding) model is judged by the proportion of brain signal variance that it can account for (Naselaris et al 2011, Kriegeskorte and Douglas 2019). However, much of brain activity is not stimulusrelated, so that proportion is small even for a model that perfectly predicted all stimulus-related brain activity. Analogous comments can be made with respect to backward models (stimulus reconstruction) that infer only select aspects of the stimulus representation rather than its entirety.

The appeal of hybrid models such as CCA (Dmochowski et al 2017, de Cheveigné et al 2018, Zhuang et al 2020) is that both stimulus and EEG are stripped of irrelevant variance, leaving remainders that can more usefully related one to the other. The model then is predictive of a transform of the measured brain response, rather than of the response itself, which makes it harder to interpret than a forward model. For example, model $\mathbf{F}$ defines a set of linear transforms of the time-lagged EEG signals, each predicted from the stimulus envelope via an FIR filter, which harder to interpret than model B that directly predicts an EEG channel, or even model $\mathbf{D}$ that predicts spatially filtered EEG.

The upside of hybrid models is that the transformed response XG (right hand side of equation (2)) arguably offers a closer (less noisy) view of the information coded by sensory-dependent parts of brain activity (Kriegeskorte and Douglas 2019). Equation (2) can be generalized to more complex transforms $f(A)$ and $g(X)$ (e.g. (Andrew et al 2013)), and it may be useful to allow $f$ to depend on $X$ (e.g. sensory processing dependent on brain state) and $g$ to depend on $A$ (e.g. a different model of brain activity in response to speech and music).

\subsection{What makes a good model?}

Prior studies using a forward model (similar to model B) or a backward model (similar to $\mathbf{C}$ or $\mathbf{E}$ ) typically report performance that is 'above chance' but still rather poor. For example, a score of $r=$ 0.1 to 0.2 means only $1 \%$ to $4 \%$ of the variance is explained, and furthermore a correct-classification score of $90 \%$ for a segment of $60 \mathrm{~s}$ duration (typical subject of the study of O'Sullivan et al (2015)), implies a decision delayed by one minute and wrong on one trial out of every ten. For applications, it is crucial to achieve shorter latency and better reliability, and from the scientific perspective it is desirable to find models that allow a better fit to the data.

CCA results in a better fit as reflected by higher values of the correlation metric (compare models $\mathbf{C}$ versus $\mathbf{D}$, or $\mathbf{E}$ versus $\mathbf{F}$ ) and the multiple correlation coefficients support multivariate classification, with a further boost to task-based metrics.

An important ingredient in the more successful models is lags, that allow the algorithms to synthesize FIR or multichannel FIR filters that can absorb convolutional mismatch between the stimulus and response, thus resulting in better performance (compare models $\mathbf{A}$ versus $\mathbf{B}, \mathbf{C}$ versus $\mathbf{D}$, or $\mathbf{E}$ versus $\mathbf{F}$ ). Adding lags increases the dimensionality of the data 
space, which is beneficial as long as the optimal transforms can be found. If not, due to overfitting, the larger dimensionality may instead by harmful.

Model overfitting was addressed here using dimensionality reduction which can be achieved trivially by discarding sensor channels (with limited success, cf dotted line in figure 5), or limiting the number of lags (with greater success, figure 6 center and right). Replacing the set of lags by a smaller number of channels of a dyadic filter bank also reduces dimensionality, with a considerable reduction in computation cost but little difference in performance (compare red and blue lines in figure 6). Applying PCA and selecting a subset of components also reduces dimensionality, with a slight boost in performance (figure 5) (see also (de Cheveigné 2021)). An additional benefit is to reduce computational cost, which can otherwise become prohibitive if many lags are introduced (the bottleneck is an eigendecomposition which costs $\left.o\left(N^{3}\right)\right)$.

The reduction in performance beyond $L=32$ ( $\sim 250 \mathrm{~ms}$ ) that we observed for this dataset (figure 6) suggested overfitting, which could merely result from a larger number of free parameters, or from the fact that higher-order FIR filters can enhance slow patterns (low frequencies) that do not generalize from training data to test. The latter seems more likely since replacing $L$ lags by a smaller number of dyadic filters of order $L$ had little impact on performance (compare blue to red in figure 6). The knee occurs at the same value of $L$ (32), suggesting that filter order (or lag span) is the critical factor.

With a $\sim 200 \mathrm{~ms}$ shift and a maximum lag of $\sim 250 \mathrm{~ms}$, the model associates stimulus samples with response samples that occur up to $\sim 450 \mathrm{~ms}$ later. However, we cannot use this to make a strong statement concerning brain processing latencies because of the potential smearing effect of the filters applied in preprocessing (section 2.3) (de Cheveigné and Nelken 2019).

\subsection{Whither now?}

Further boosts in performance are needed to enhance the feasibility of potential applications. Based on what we know so far, there are several directions worth pursuing.

One is to improve the stimulus representation. Here, we used a rather crude representation, the stimulus envelope. Richer representations such as auditory filterbank (Biesmans et al 2017), higher-order linguistic structure (Di Liberto et al 2015), onsets (Oganian and Chang 2019), or voice pitch (Forte et al 2017, Teoh et al 2019), etc have been explored but remain to be developed further and integrated. Multiset CCA (MCCA), which allows merging EEG across subjects, may ease development of such stimulus representations (de Cheveigné et al 2019).

A second direction is to extract more information from the brain response. Typical models (including those reported here) exploit low-frequency components, but useful information may also be carried by high-frequency power (Forte et al 2017, Teoh et al 2019, Synigal et al 2020). Standard linear techniques (such as CCA) are not directly applicable to enhance weak sources of power, but it may be possible to use quadratic component analysis (QCA) for that purpose (de Cheveigné 2012). This entails forming crossproducts between channels and/or lags, leading to very high-dimensional data for which an appropriate dimensionality-reduction strategy is crucial.

A third direction is better integration of information over time. As figure 7 (top) shows, errors occur only for segments for which the mismatch $d_{m}$ is large, and these occupy only a small fraction of the time axis. A better understanding of what triggers largemismatch events might allow them to be mitigated. Alternatively, since they are flagged by a high value of $d_{m}$, it may be possible to integrate over a highreliability (low $d_{m}$ ) context to offer the application a reliable decision.

A fourth direction is more prosaic: better preprocessing, filtering, artifact rejection, parameter tuning, etc. Performance metrics are sensitive to preprocessing parameters, but no attempt was made to tune them in this study.

Finally, a fifth direction is to use more recent machine-learning methods in lieu of expertise-based approaches, in the faith that they will discover the same regularities and structure as embodied by handcrafted methods, and more. Results so far are modest (Ciccarelli et al 2019, Das et al 2020, Jalilpour Monesi et al 2020, Tian and Ma 2020), but success in other fields suggests that machine-learning approaches are well worth pursuing.

\section{Data availability statement}

The data that support the findings of this study are openly available at the following URL/DOI: http:// doi.org/10.5281/zenodo.3618205.

\section{Acknowledgments}

This work was supported by Grant Nos. ANR-10LABX-0087 IEC, ANR-10-IDEX-0001-02 PSL, and ANR-17-EURE-0017. Jens Hjortkjaer and Søren A Fuglsang were supported by the Novo Nordisk Foundation synergy Grant No. NNF17OC0027872 (UHeal). We appreciate many helpful discussions with Jonathan Berent and his team. We gratefully acknowledge useful comments from two reviewers on earlier versions of the manuscript.

\section{ORCID iDs}

Alain de Cheveigné (1) https://orcid.org/0000-00027305-1891 
Malcolm Slaney (1) https://orcid.org/0000-00019733-4864

\section{References}

Ahissar E, Nagarajan S, Ahissar M, Protopapas A, Mahncke H and Merzenich M M 2001 Speech comprehension is correlated with temporal response patterns recorded from auditory cortex Proc. Natl Acad. Sci. 98 13367-72

Aiken S J and Picton T W 2008 Human cortical responses to the speech envelope Ear and Hear. 29 139-57

Akbari H, Khalighinejad B, Herrero J L, Mehta A D and Mesgarani N 2019 Towards reconstructing intelligible speech from the human auditory cortex Sci. Rep. 9874

Andrew G, Arora R, Bilmes J and Livescu K 2013 Deep canonical correlation analysis Proc. 30th Int. Conf. on Machine Learning p 9 (https://proceedings.mlr.press/v28/andrew13.html)

Arandjelović R and Zisserman A 2018 Objects that Sound Computer Vision-ECCV 2018 vol 11205, ed V Ferrari, M Hebert, C Sminchisescu and Y Weiss (Berlin: Springer) pp 451-66 (https://openaccess.thecvf.com/content_ECCV_ 2018/papers/Relja_Arandjelovic_Objects_that_Sound_ ECCV_2018_paper.pdf)

Bednar A and Lalor E C 2020 Where is the cocktail party? Decoding locations of attended and unattended moving sound sources using EEG NeuroIm-age 205116283

Biesmans W, Das N, Francart T and Bertrand A 2017 Auditory-inspired speech envelope extraction methods for improved EEG-based auditory attention detection in a cocktail party scenario IEEE Trans. Neural Syst. Rehabil. Eng. 25 402-12

Broderick M P, Anderson A J and Lalor E C 2019 Semantic context enhances the early auditory encoding of natural speech J. Neuro-science 39 7564-75

Ciccarelli G, Nolan M, Perricone J, Calamia P T, Haro S, O'Sullivan J, Mesgarani N, Quatieri T F and Smalt C J 2019 Comparison of two-talker attention decoding from EEG with nonlinear neural networks and linear methods Sci. Rep. 911538

Crosse M J, Di Liberto G M, Bednar A and Lalor E C 2016 The multivariate temporal response function (mTRF) toolbox: a MATLAB toolbox for relating neural signals to continuous stimuli Front. Hum. Neurosci. 10604

Das N, Zegers J, Van hamme H, Francart T and Bertrand A 2020 Linear versus deep learning methods for noisy speech separation for EEG-informed attention decoding J. Neural Eng. 17046039

de Cheveigné A 2012 Quadratic component analysis NeuroImage $593838-44$

de Cheveigné A 2021 Shared component analysis NeuroImage 226117614

de Cheveigné A and Arzounian D 2018 Robust detrending, rereferencing, outlier detection and inpainting for multichannel data NeuroImage 172 903-12

de Cheveigné A, di Liberto G M, Arzounian D, Wong D, Hjortkjaer J, Asp Fuglsang S and Parra L C 2019 Multiway canonical correlation analysis of brain signals NeuroImage $186728-40$

de Cheveigné A and Nelken I 2019 Filters: when, why and how (not) to use them Neuron 102 280-93

de Cheveigné A and Parra L C 2014 Joint decorrelation, a versatile tool for multichannel data analysis NeuroImage 98 487-505

de Cheveigné A, Wong D, Di Liberto G, J H, M S and Lalor E 2018 Decoding the auditory brain with canonical component analysis NeuroImage 172 206-16

Decruy L, Vanthornhout J and Francart T 2020 Hearing impairment is associated with enhanced neural tracking of the speech envelope Hear. Res. 393107961

Di Liberto G M, O’Sullivan J A and Lalor E C 2015 Low-frequency cortical entrainment to speech reflects phoneme-level processing Curr. Biol. 25 2457-65
Ding N and Simon J Z 2012 Neural coding of continuous speech in auditory cortex during monaural and dichotic listening $J$. Neurophysiol. 107 78-89

Dmochowski J, Ki J, DeGuzman P, Sajda P and Parra L 2017 Extracting mutlidimensional stimulus-response correlations using hybrid encoding-decoding of neural activity NeuroImage 180 134-46

Forte A E, Etard O and Reichenbach T 2017 The human auditory brainstem response to running speech reveals a subcortical mechanism for selective attention eLife e27203

Fuglsang S A, Dau T and Hjortkjjær J 2017 Noise-robust cortical tracking of attended speech in real-world acoustic scenes NeuroImage 156 435-44

Fuglsang S A, Märcher-Rørsted J, Dau T and Hjortkjær J 2020 Effects of sensorineural hearing loss on cortical synchronization to competing speech during selective attention J. Neurosci. 40 2562-72

Goossens T, Vercammen C, Wouters J and van Wieringen A 2018 Neural envelope encoding predicts speech perception performance for normal-hearing and hearing-impaired adults Hear. Res. 370 189-200

Hausfeld L, Riecke L, Valente G and Formisano E 2018 Cortical tracking of multiple streams outside the focus of attention in naturalistic auditory scenes NeuroImage 181 617-26

Hillyard S A, Hink R F, Schwent V L and Picton T W 1973 Electrical signs of selective attention in the human brain Science 182 177-80

Jaeger M, Mirkovic B, Bleichner M G, Debener S 2020 Decoding the attended speaker from EEG using adaptive evaluation intervals captures fluctuations in attentional listening Frontiers Neurosci. 14603

Jalilpour Monesi M, Accou B, Montoya-Martinez J, Francart T and Van hamme H 2020 An LSTM based architecture to relate speech stimulus to EEG (IEEE) pp 941-5

Kerlin J R, Shahin A J and Miller L M 2010 Attentional gain control of ongoing cortical speech representations in a 'cocktail party' J. Neurosci. 30 620-8

Kriegeskorte N and Douglas P K 2019 Interpreting encoding and decoding models Curr. Opin. Neurobiol. 55 167-79

Kubanek J, Brunner P, Gunduz A, Poeppel D and Schalk G 2013 The tracking of speech envelope in the human cortex PLoS One 8 e53398

Lalor E C, Power A J, Reilly R B and Foxe J J 2009 Resolving precise temporal processing properties of the auditory system using continuous stimuli J. Neurophysiol. $102349-59$

Mesgarani N and Chang E F 2012 Selective cortical representation of attended speaker in multi-talker speech perception Nature 485 233-6

Molloy K, Griffiths T D, Chait M and Lavie N 2015 Inattentional deafness: visual load leads to time-specific suppression of auditory evoked responses J. Neurosci. 35 16046-54

Monesi M J, Accou B, Montoya-Martinez J, Francart T and Hamme H V 2020 An LSTM based architecture to relate speech stimulus to EEG ICASSP pp 941-5

Montoya-Martínez J, Vanthornhout J, Bertrand A and Francart T 2021 Effect of number and placement of eeg electrodes on measurement of neural tracking of speech PLoS One 16 e 0246769

Murphy K P 2021 Probabilistic Machine Learning: An Introduction (Cambridge, MA: MIT Press)

Naselaris T, Kay K N, Nishimoto S and Gallant J L 2011 Encoding and decoding in fMRI NeuroImage 56 400-10

O'Sullivan J A, Power A J, Mesgarani N, Rajaram S, Foxe J J, Shinn-Cunningham B G, Slaney M, Shamma S A and Lalor E C 2015 Attentional selection in a cocktail party environment can be decoded from single-trial EEG Cerebral Cortex 25 1697-706

O'Sullivan J, Herrero J, Smith E, Schevon C, McKhann G M, Sheth S A, Mehta A D and Mesgarani N 2019 Hierarchical encoding of attended auditory objects in multi-talker speech perception Neuron 104 1195-1209.e3 
Oganian Y and Chang E F 2019 A speech envelope landmark for syllable encoding in human superior temporal gyrus $\mathrm{Sci}$. Adv. 14

Owens A and Efros A A 2018 Audio-Visual Scene Analysis with Self-Supervised Multisensory Features Computer Vision ECCV 2018 vol 11210, V Ferrari, M Hebert, C Sminchisescu and Y Weiss Series Title: Lecture Notes in Computer Science (Berlin: Springer) pp 639-58 (https://openaccess.thecvf. com/content_ECCV_2018/papers/Andrew_Owens_AudioVisual_Scene_Analysis_ECCV_2018_paper.pdf)

Power A J, Lalor E C and Reilly R B 2011 Endogenous auditory spatial attention modulates obligatory sensory activity in auditory cortex Cereb. Cortex 21 1223-30

Puvvada K C and Simon J Z 2017 Cortical representations of speech in a multitalker auditory scene J. Neurosci. 37 9189-96

Scheer M, Bülthoff H H and Chuang L L 2018 Auditory task irrelevance: a basis for inattentional deafness Hum. Factors: J. Hum. Factors Ergon. Soc. 60 428-40

Synigal S R, Teoh E S and Lalor E C 2020 Including measures of high gamma power can improve the decoding of natural speech from EEG Front. Hum. Neurosci. 14130
Teoh E S, Cappelloni M S and Lalor E C 2019 Prosodic pitch processing is represented in delta-band EEG and is dissociable from the cortical tracking of other acoustic and phonetic features European J. Neurosci. 50 3831-42

Tian Y and Ma L 2020 Auditory attention tracking states in a cocktail party environment can be decoded by deep convolutional neural networks J. Neural Eng. 17036013

Tibshirani S, Friedman H and Hastie T 2017 The Elements of Statistical Learning Series in Statistics (New York: Springer)

Treder M S, Purwins H, Miklody D, Sturm I and Blankertz B 2014 Decoding auditory attention to instruments in polyphonic music using single-trial EEG classification J. Neural Eng. 11026009

Wong D D E, Fuglsang S A, Hjortkjær J, Ceolini E, Slaney M and de Cheveigné A 2018 A comparison of regularization methods in forward and backward models for auditory attention decoding Front. Neurosci. 12531

Zhuang X, Yang Z and Cordes D 2020 A technical review of canonical correlation analysis for neuroscience applications Hum. Brain Mapp. 41 3807-33

Zuk N J, Teoh E S, Lalor E C 2020 EEG-based classification of natural sounds reveals specialized responses to speech and music NeuroImage 210116558 